(C) 2018 IEEE. Personal use of this material is permitted. Permission from IEEE must be obtained for all other uses, in any current or future media, including reprinting/republishing this material for advertising or promotional purposes, creating new collective works, for resale or redistribution to servers or lists, or reuse of any copyrighted component of this work in other works. 


\title{
RESCUE: Cross-Sectoral PhD Training Concept for Interdependent Reliability, Security and Quality
}

\author{
Heinrich Theodor Vierhaus ${ }^{1}$, Maksim Jenihhin ${ }^{2}$, Matteo Sonza Reorda ${ }^{3}$ \\ ${ }^{1}$ Brandenburg University of Technology, Germany, heinrich.vierhaus@b-tu.de \\ ${ }^{2}$ Tallinn University of Technology,Estonia, maksim@ati.ttu.ee \\ ${ }^{3}$ Politecnico di Torino, Italy, matteo.sonzareorda@polito.it
}

\begin{abstract}
The recently started European Training Network (ETN) RESCUE advances scientific competences in the demanding and mutually dependent aspects of nano-electronic systems design, i.e. reliability, security and quality, as well as related electronic design automation tools. Second, it provides early-stage researchers with innovative cross-sectoral training in the involved disciplines and beyond, preparing them to face today's and future challenges in nano-electronics design. Furthermore, they are also trained to be innovative, creative, and more important - will have an entrepreneurial mentality. The latter will help to compile ideas into products and services for economic and social benefits and creates qualified workforce and knowledge for the industry. The consortium consists of leading European research groups competent to tackle the interdependent challenges in a holistic manner, and is excellently balanced in terms of academic and industrial training and research facilities.
\end{abstract}

Keywords - PhD training, nano-electronic systems design, H2020 MSCA ITN.

\section{INTRODUCTION}

In the IoT Era, electronic systems, ultimately, represent the physical backbone of our increasingly digital world. They are being deployed in life-critical application domains, such as healthcare, transportation, automotive and security, serving societal needs in Europe. Here, the impact and consequences of in-field failures, security attacks, or hardware defects could be catastrophic. Reliability, quality and security cannot be treated anymore as standalone aspects and have inherent trade-offs with application constraints, cost-efficiency, energy consumption, performance of the system, and its safety requirements. In order to underpin the next generation implementation technologies and rescue the steady growth of nano-electronic systems' performance, new concepts, methodologies and tools for interdisciplinary and multi-scale design are urgently needed.

Along with ambitious research goals, the Marie Skłodowska-Curie action H2020 RESCUE ITN ETN [1] provides an innovative interdisciplinary training for future European engineers and researchers, placing strong emphasis on developing their creative and entrepreneurial mentality. Traditionally, the research and training in Europe for these highly interdependent challenges in nano-electronic system design is fragmented and performed by scattered communities. RESCUE has an excellently balanced cross-sectoral consortium of reputable leading European research groups and companies competent to tackle the reliability, security and quality aspects in a holistic manner.
The industrial sector behind this initiative includes innovative and award-winning European SMEs from the areas of nano-electronics reliability and security - IROC Technologies and Intrinsic-ID. The large companies on board are Cadence, the global electronic design automation company, and Robert Bosch, the European automotive electronics flagship. The latter supports the ETN as a partner organization. As cutting-edge research institution, Leibniz-Institute IHP serves as a bridge for knowledge transfer between the sectors. The academic sector is represented by Delft, Brandenburg and Tallinn Universities of Technology, and Politecnico di Torino. Seven of eight beneficiaries (from both academic and private sector) have recruited two early stage researchers (ESRs) each for 3 years and one industrial beneficiary has recruited one ESR.

RESCUE was launched on April 1, 2017 and will last for 4 years with the total budget 3.76 MEUR, as a contribution by European Commission.

The rest of this paper is organized as follows: Section II discusses challenges in the $\mathrm{PhD}$ education focusing on the Computer Engineering domain, Sections III and IV introduces the RESCUE training concept emphasizing the cross-sectoral aspect and research collaboration, Section V summarizes the current status and Section VI draws the conclusions.

\section{Challenges in Computer EngineERING PhD EDUCATION}

Making academic researchers fit for not only an academic career but also for qualified employment in industries has been a matter of concern for quite some time [2],[3],[4],[5],[6]. Having academics work in a purely research-oriented environment, where applicability of results to real-life problems is of little concern, is risky for young people at best. Experience was gained in networking projects such as DAAD funded DCPS (Dependable Cyber Physical Systems), involving universities in 4 countries, IHP as a research lab and industries as associate partners [2]. It showed that the development of academic and industrial skills in young people in combination requires a new educational concept, which is different from normal academic $\mathrm{PhD}$ education. The basic structure of traditional Central European (non-) education towards a doctoral grade is shown in Fig. 1. Typically, doctoral candidates would enter employment as teaching- and research assistants and start finding out about their area of interest and the relevant state of the art under guidance of a single professor. Practical relevance of the research done thereafter was pretty much dependent of specific knowledge, interest and scientific connections of a single 
supervising professor. Hence the doctoral candidate was typically very much dependent on this person, often without much of a chance to start her / his own scientific networking.

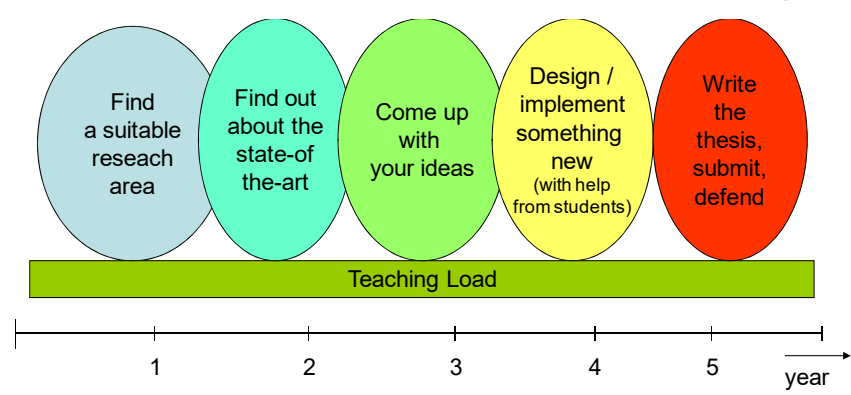

Fig. 1 Traditional scientific (non-) education

With a total duration of about 5 years (at the average), doctoral students typically were in their mid-30s, when suddenly having to find a new job outside the university. Without being prepared for this situation, handling it was complicated at best. The type of education planned for RESCUE is shown in Fig. 2. Typically, the time slot is about 3 years rather than 5 years.

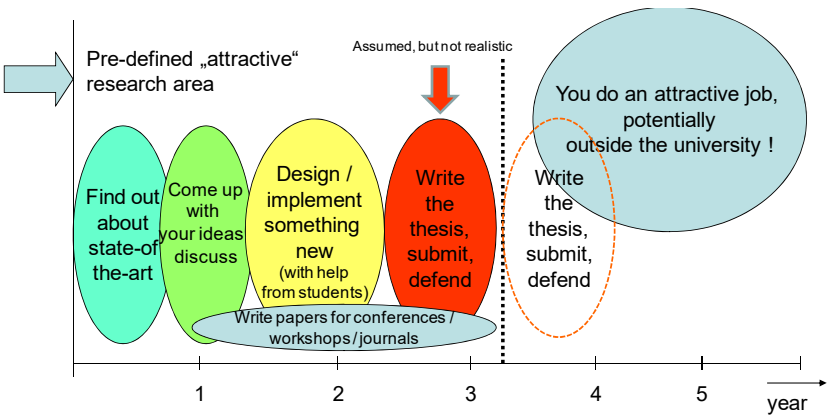

Fig. 2 RESCUE scientific education

First, new early stage researchers get a strong orientation at the beginning. Getting active in research and producing paper has to start very early. But during this active phase, there must be a strong interaction with academic and industrial partners for broader orientation and personal networking. Common measures of education within the RESCUE community broaden the view and promote the development towards the status of a senior scientist. Experienced professors are quite aware that orientation, individual research, broader orientation, and thesis writing will possibly not fit into a time slot of just 3 years. For keeping existing European standards in doctoral education, therefore we anticipate that a candidate will have done all her /his essential parts of research and may start writing a thesis from then.

\section{RESCUE TRAINING CONCEPT}

To address challenge of cross-sectoral interdisciplinary training, RESCUE ETN has introduced a novel concept of training for "early stage researchers" (ESRs) based on four pillars of activities (see Fig. 3).

The first pillar Research training through individual research projects of ESRs is a training-through-research activity. This starts with an orientation phase at the host institution. Here, the ESR gets familiar with the training organization aspects and adjusts his or her technical and soft skills background. As experience shows, new ESRs may need to participate in masterlevel courses as well, since often their own master level education is basically matching, but not totally adequate. Next, this pillar covers training by focused research setting Individual Research Projects along with cross-sectoral secondments of ESRs to other project partners. This allows acquiring a broader view, establishing personal working contacts, including with other RESCUE ESRs, and getting close to practical industrial working styles and real-life problems.

The second pillar Complementary scientific training is education by common RESCUE events. First, it is the series of annual internal RESCUE Winter Workshops which offers a wide range of tutorials for ESRs (the first one was organized by BTU in Cottbus, Germany, in December 2017). This series started with tutorials of an introductory type in 2017 and will get more advanced in later years. From 2018, this is also a forum where ESRs will report about their own work and discuss ideas, approaches and problems with their colleagues and all participating experts from industry and academia. Second, it is a series of summer school events "BELAS - Biannual European - Latin American Summer School on Design, Test and Reliability" [7], which are organized annually in European summer (with a matching partner-event of the same series in Latin America organized during summer in the Southern Hemisphere). This series of workshops supplies advanced tutorials in the area of dependable and secure computer-based

Training through Pillars 2-4 : events, soft skills and local curricula

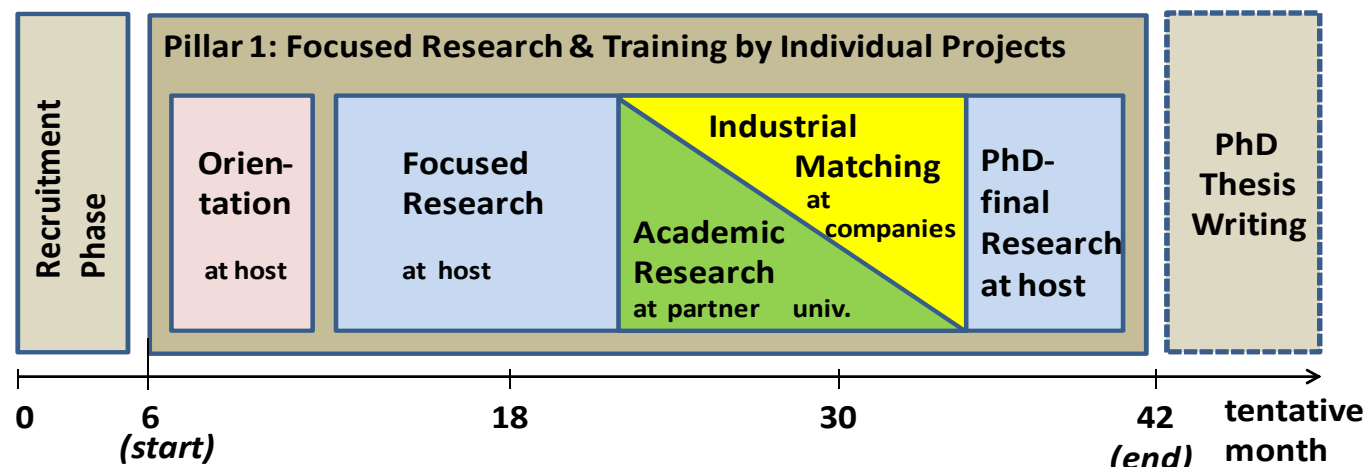

Fig. 3. RESCUE ETN training concept 
systems, but also offers sessions where students present their own work (at PhD Forum). BELAS is open to participation by external PhD students from Europe and beyond and therefore establishes a wider networking opportunities for RESCUE ESRs.

The third pillar Complementary transferable skills training consists of courses, e.g. in the social area, on management skills, and on scientific capabilities such as paper writing and thesis writing. Such capabilities may be obtained at the consortium level by introductory courses at workshop events, but more in-depth also from in-house training at the respective home universities. The internal RESCUE Winter Workshops also cover non-technical topics such as getting started in research, paper writing and thesis writing.

The fourth pillar Additional training opportunities provided by existing curricula of partners implies lectures, labs, and seminars, typically specific events for doctoral students, mostly provided by universities [8], [9], [10], [11]. ESRs hosted by industrial partners participate in such courses upon demand. At the same time, RESCUE industrial partners have an established portfolio of training events including the program by Cadence Academic Network [12]. As the previous backgrounds of ESRs when starting their employment as ESRs differ a lot, additional background (and training by labs) from existing master programs and $\mathrm{PhD}$ programs is needed. These activities are supported on a wide scale by an "information turn-table", which makes material from specific master- and doctoral level courses available to all interested ESRs upon demand.

First experience during the start-up phase of RESCUE has shown that this scheme may get an extension from the start-up phase of the project. Typically, universities within their own graduate research schools, offer specific 1-3 day intensive courses on, for example, paper writing and thesis writing. As far as possible, such programs in specific partner universities will be also opened upon demand for $\mathrm{PhD}$ students and young engineers from RESCUE consortium.

Typically, programs for doctoral studies existing at partner universities include examinations of various types. Dedicated educational activities in RESCUE also require a mechanism of feedback from participating students including a mechanism that may let them earn credit points. For such purpose, lecturers of tutorials during the RESCUE Winter Workshop composed a catalogue of questions for participating students. Evaluation was done using existing web-based tools that support "remote" examinations. As the first implementation showed positive results with respect to the degree of active participation and the quality of questions and answers, this mechanism will be used for future workshops as well.

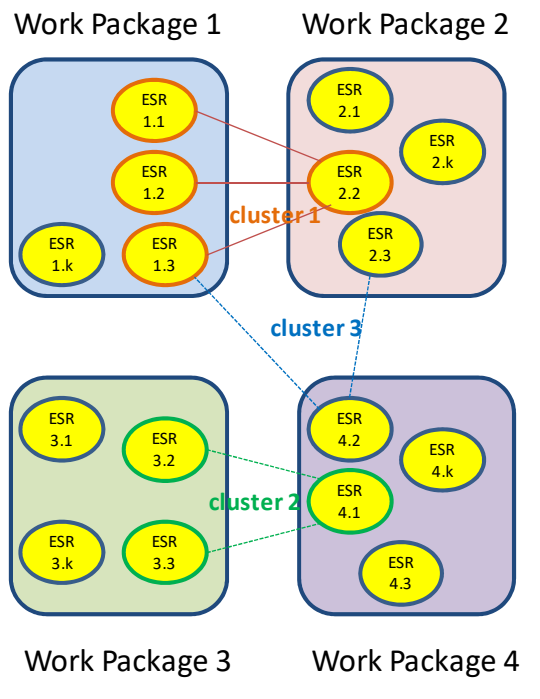

Fig 4. The concept of research clusters

\section{AdVANCED TRAINING CONCEPTS AND NETWORKING}

\section{A. Research Clusters}

First experience in RESCUE has shown that research topics of ESRs are strongly correlated, also across the borders of the pre-defined technical work packages: WP1 Reliability, WP2 Quality, WP3 Security, WP4 EDA Tools and Methodologies. Hence it is potentially useful to organize research clusters for ESRs that work in closely related areas (see Fig. 4). They should be able to exchange documents, specifically white papers and preliminary results openly. Such clusters should optionally also include other young researchers from partner universities, who

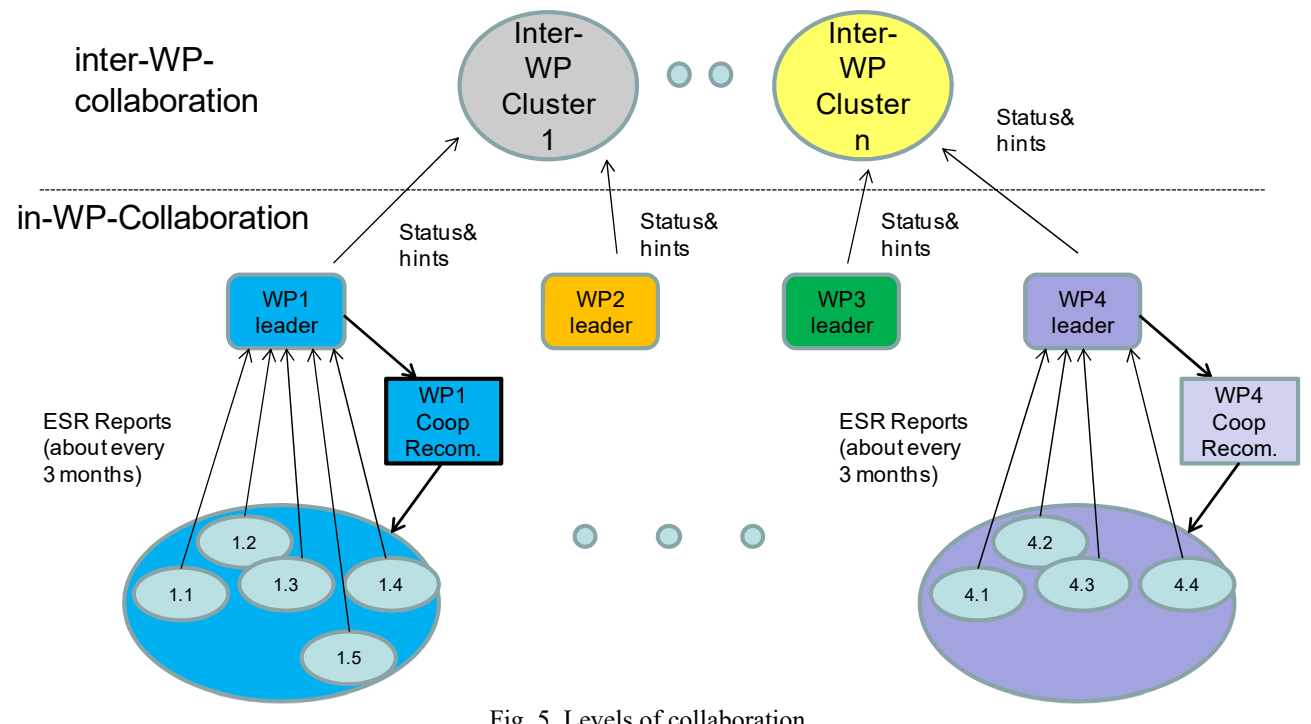

Fig. 5. Levels of collaboration 
are not supported by RESCUE funding. Currently, 1-3 research clusters are being established, each with a university professor or an industrial expert to act as a leader.

Consider Fig. 5. A fist level of collaboration will be established at the level of the pre-defined work packages. Here the work package leader is to have an overview about the status of education and research for the respective ESRs from regular short reports. Collaboration in clusters above the work packages can then be installed upon demand. For example, design tool environments developed in WP4 EDA Tools and Methodologies may incorporate specific design and test tools developed elsewhere and at the partner institutions [13]. Furthermore, a high-level functionality monitoring the fault / error status of a system at the operation systems level may use low-level faultrelated information, i.e. from ESRs in WP1 Reliability.

The main effect that can be expected from research clusters is beginning collaboration of ESRs from the start-up phase, for example also by exchanging educational materials for common use. In later phases of the project, research clusters should yield joint publication from several ESRs. Even a scheme of mutual proof-reading of publications would be supported by such approach. We expect that, after the definition of research clusters specific electronic communication platforms will be established, supported by the respective leaders.

\section{B. RECUE Turn Table knowledge pool}

RESCUE training events will yield a large number of tutorials, which should be made available for downloading and personal re-use by the ESRs, but also for other doctoral students and engineers from the consortium. Beyond a collection of tutorials from RESCUE events, this knowledge pool may also include doctoral- and master-level educational materials from all partner universities.

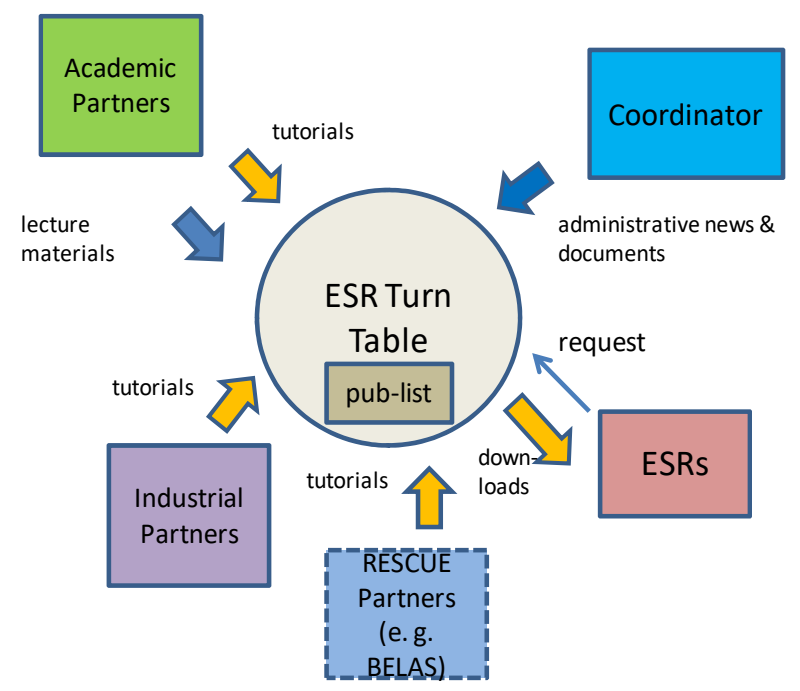

Fig. 6. RESCUE ESR Information Turn Table

Fresh ESRs should be able to post their request for educational materials that they need and either get from lectures beyond their home university of from other sources like books and overview articles in journals. The turn-table is also used to distribute administrative information to all ESRs and to provide the whole RESCUE training network community with updated information on recent publications from partners. The variety of information that targeted for RESCUE Turntable is depicted in Fig. 7.

\section{Extended industrial training}

Getting industrial partners involved into RESCUE was an essential part of the concept from the beginning also considering

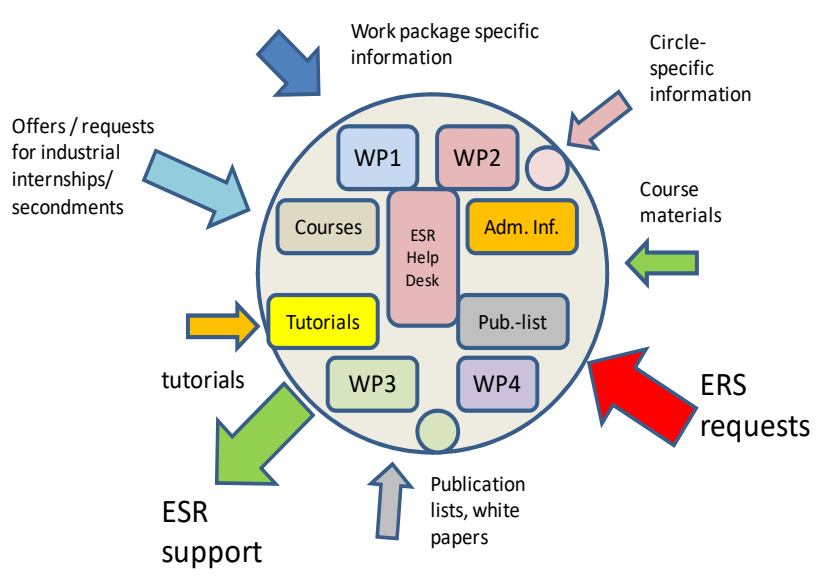

Fig. 7. Information Turn Table, advanced status

the EU policies [14], [15]. Making $\mathrm{PhD}$ students fit for an industrial career is not an easy task, since companies work in very different manners. Industrial links are easiest to establish towards small innovative companies that were themselves founded out of academic research groups and which keep research links into academia as some sort of a life-line. In the RESCUE consortium, we can identify IROC S.A. of Grenoble / France and Intrinsic ID N. V. of Eindhoven / Netherlands as such companies. They are also the most likely companies to hire recent $\mathrm{PhDs}$ later. Companies working in the area of electronic design automation (EDA) typically keep good links into academia in order to become aware of the latest academic developments in algorithms as soon as possible. Therefore, also Cadence Design Systems became a natural partner for RESCUE from the start. All these partners employ their own early stage researchers (ESRs), who will formally also be hosted and enrolled by one of the partner universities.

Except for this direct link, also ESRs from other academic partners will take visits to such companies for networking and exchange of research results.

Also in the past, it has been much more difficult to get large electronic hardware companies involved into ventures such as RESCUE. One reason is that research results, implemented in software tools and algorithms or in innovative concepts of circuit and system design, will find an application in products like electronic motor control only indirectly and with larger delays. Furthermore, most of hardware developments are strongly secured by patents, which academia is typically not so familiar with. Last, companies such as Robert Bosch GmbH operate their own large-scale research departments, which outnumber the researchers in the RESCUE consortium by far. Hence, such companies typically look for academic contacts when a sudden event happens, such as hackers intruding automotive control [16], for which fast solutions must be found. In such cases, a company starts a new project which is still not directly connected with ongoing system developments and can therefore easier host academic guests. Hence, Robert Bosch $\mathrm{GmbH}$ is 
TABLE I. CROSS-SECTORAL SUPERVISION ARRANGEMENTS FOR RESCUE ETN EARLY-STAGE RESEARCHERS

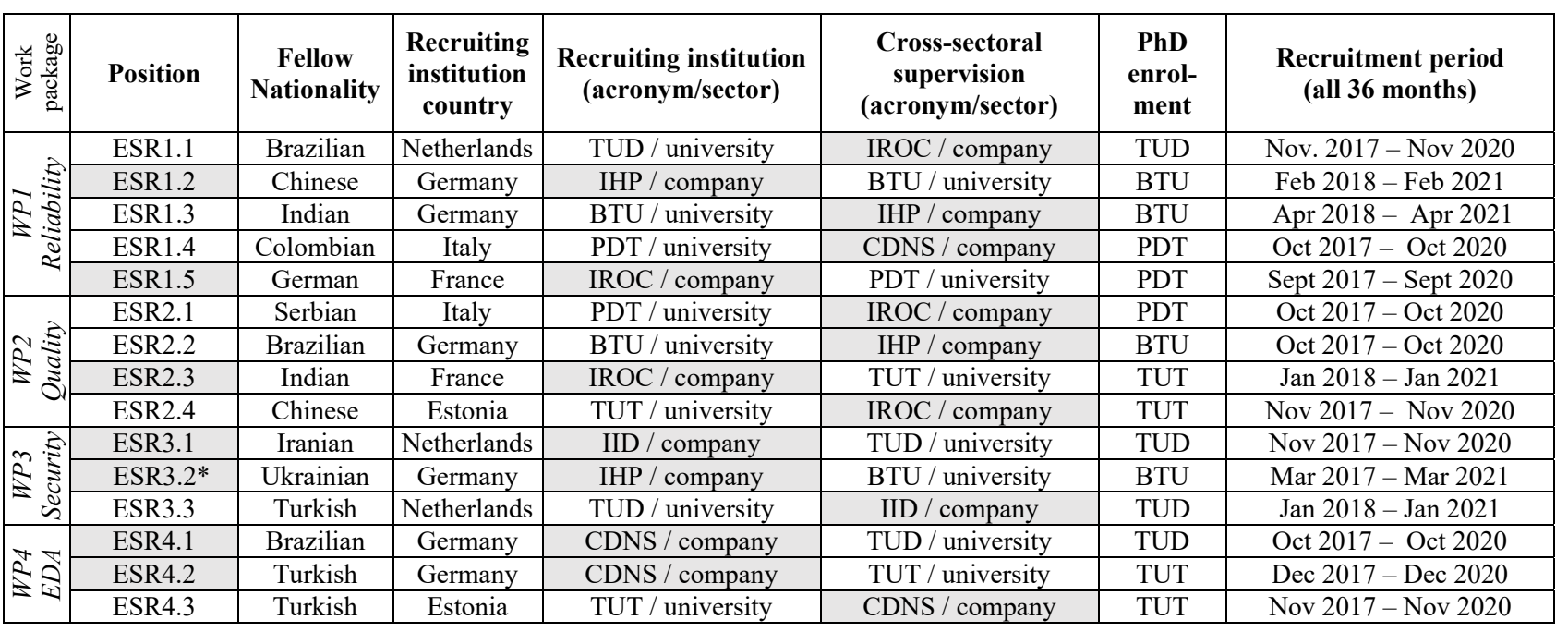

* This ESR position is also supported by research supervision by Robert Bosch GmbH as a Partner Organization in the ETN

involved as a partner organization in RESCUE, providing industrial internships for an ESR who works in the area of hardware security.

Essential for the success of RESCUE therefore is a partner that has both an academic and an industrial "leg" such as the Leibniz-Institute for Innovative Microelectronics (IHP GmbH). Such institutes, with a strong involvement into industrial research and development projects, are typically aware of industrial constraints such as patent issues, but they also keep strong academic links by joint labs with universities. Notably, recent $\mathrm{PhDs}$ from institutes like IHP seem to have a relatively high chance to be hired by also large electronic companies, because they are scientifically productive and innovative, but also familiar with industrial practice. Hence, having IHP on board in RESCUE, also for secondments of ESRs from academic partners will play a major role towards the success of the project.

\section{CURRENT STATUS}

The RESCUE project started on April 1, 2017. During the first year, major activities included, first of all, hiring the expected ESRs. The Call for applicants was widely distributed and more than 130 applications were submitted. Interestingly, only 19 of them came from EU and associated countries. Selection of the candidates was performed taking into account the project constraints and resulted in the identification of the 15 winners. A detailed individual career development plan was identified for each of them.

Secondly, the partners started the training activities, according to the principles introduced in the previous Sections. Besides local training provided by individual partner institutions, ESRs are attending some international training events in their areas of interest, such as schools or tutorials in the frame of international conferences. Moreover, a first internal event was organized, where all the ESRs and their tutors met. During the same event a number of tutorials were offered to ESRs by experts from the partner institutions, focusing both on technical topics and on soft skills. A key aspect of the event was the cross-sectoral emphasis. In particular, there was a strong support from the Cadence including special webinars on industrial practices organized for the RESCUE network. Later, the first RESCUE BELAS summer school event [7] was organized in collaboration with international research community that was open for participation by external students and researchers.

The status of cross-sectoral supervision arrangements for the fellows recruited for the RESCUE ETN ESR positions is presented in Table I.

\section{CONCLUSIONS}

In previous projects on doctoral education across national borders [2], we offered a broader scope on top of traditional doctoral education schemes, based mainly on tutorial workshops involving international experts. Taking a look at successful (now) doctors from TU Tallinn and BTU-CS, who were most eager to get a broader insight on dependable hardware / software systems, the results are promising. In some cases, the wider scope of knowledge beyond the "deep drill" in their personal research was at least helpful if not essential for immediate industrial employment after the end of the university contracts. Including external professors into the respective doctoral examination procedures helped to secure high quality. RESCUE goes a step further by re-organizing the doctoral education scheme from the start. The purpose is to keep (at least) the scientific level of students and their work, while giving them the essential overview to become "senior scientists" with excellent prospects both for industrial employment and for an academic career. Given the time frame of only 3 years for the essential phase of scientific evolution, RESCUE takes a risky venture at least. But, with formal doctoral education at a level hardly above the traditional master becoming fashionable elsewhere, keeping European standards at the level where a doctor of $\mathrm{PhD}$ reaches the ability of conducting self-organized research seems to be worth the effort.

\section{ACKNOWLEDGMENTS}

This work was supported in part by H2020 MSCA ITN RESCUE that has received funding from the European Union's 
Horizon 2020 research and innovation programme under the Marie Skłodowska-Curie grant agreement No 722325, by H2020 TWINN TUTORIAL, and by the Estonian Ministry of Education and Research institutional research grant IUT19-1.

\section{REFERENCES}

[1] RESCUE ETN website [http://rescue-etn.eu/]

[2] Heinrich T. Vierhaus, Mario Schölzel, Jaan Raik, Raimund Ubar, "Advanced Technical Education in the Age of Cyber Physical Systems", Proc. 10th European Workshop on Microelectronics Education (EWME 2014), Tallinn, May 2014, INSPEC Accession Number: 14526779

[3] Powell, Kendall. "The future of the postdoc." Nature. 2015 Apr 9; 520 (7546):144-7. doi: 10.1038/520144a.

[4] Nurse P, Sunami A, Polka J, et al. 2015, STEM education: To build a scientist. Nature. 2015, vol.523: 371-373. doi: 10.1038/nj7560-371a

[5] Researcher Development Framework, VITAE Careers Research and Advisory Centre (CRAC), 2010 [http://www.vitae.ac.uk/rdf]

[6] Principles for Innovative Doctoral Training, EC, 2011, [http://ec.europa.eu/euraxess/pdf/research policies/Principles for Innov ative Doctoral Training.pdf]

[7] BELAS Summer School website [http://belas-event.org]

[8] TUT Curricula (Faculty of Information Technology) at [http://ois.ttu.ee/]; [http://www.ttu.ee/studying/]
[9] BTU Curricula, Faculty 1 Mathematics, Natural Sciences and Computer Science [http://www.tu-cottbus.de/fakultaet1/en/studies/programms.html]

[10] TUD Dept. of Computer Engineering curricula at [http://www.ce.ewi.tudelft.nl/education/courses/]

[11] PDT Dept. of Control and Comp. Eng. curricula [https://didattica.polito.it/pls/portal30/sviluppo.scudo new.dott?li=EN\& cod $=204 \&$ sez $=$ Course $\% 20$ curricula $]$

[12] Cadence Academic Network: [http://www.cadence.com/support/Pages/academic_network.aspx]

[13] A. Tšepurov, G. Bartsch, R. Dorsch, M. Jenihhin, J. Raik and V. Tihhomirov, "A scalable model based RTL framework zamiaCAD for static analysis," IEEE/IFIP VLSI-SoC, Santa Cruz, USA, 2012, 171-176.

[14] European University Association (2009) „Collaborative Doctoral Education: University-Industry Partnerships for Enhancing Knowledge Exchange"

[http://www.eua.be/fileadmin/user upload/files/Publications/DOCCAREERS.pdf]

[15] „Mobility of Researchers between Academia and Industry. 12 Practical Recommendations“, European Commission, 2006 [http://ec.europa.eu/euraxess/pdf/research policies/mobility of research ers light.pdf]

[16] Hackers Remotely Kill a Jeep on the Highway - With Me in It, WIRED, [https://www.wired.com/2015/07/hackers-remotely-kill-jeep-highway/] 